\title{
THE RENAL REGULATION OF ACID-BASE BALANCE IN MAN. I. THE NATURE OF THE MECHANISM FOR ACIDIFYING THE URINE ${ }^{1}$
}

\author{
By R. F. PITTS, W. D. LOTSPEICH, W. A. SCHIESS, AND J. L. AYER \\ WITH THE TECHNICAL ASSISTANCE OF PHYLLIS MINER \\ (From the Department of Physiology, Syracuse University College of Medicine, \\ Syracuse, $N . Y$.)
}

(Received for publication August 29, 1947)

Under normal conditions the urine is more acid in reaction than the plasma from which it is formed, and some 100 to $300 \mathrm{ml}$. of one-tenth normal alkali are required to titrate the 24 -hour specimen to $\mathrm{pH} 7.4$ (1). When the metabolic acid load on the body is increased in diabetic ketosis, as much as $1200 \mathrm{ml}$. of one-tenth normal titratable acid may be excreted per day (2). Since the excretion of acid in free titratable form makes available to the body an equivalent quantity of fixed base for conversion into bicarbonate, the renal mechanisms involved in acidifying the urine play a significant role in stabilizing the alkali reserve (3).

There are obviously 2 ways in which acid might gain access to the urine. First, it might be present as free buffer acid in the glomerular filtrate. If during passage of the filtrate through the renal tubules, alkaline buffer components were preferentially reabsorbed, the reaction of the urine would shift toward the acid side. Second, the acid might be added to the filtrate by some active transport mechanism resident in the renal tubular cells. The 4 possible permutations of these 2 theories are presented in diagrammatic form in Figure 1.

Only 2 acids exist in the plasma in significant quantities in freely filtrable form: monobasic phosphate and carbonic acid. Each, associated with its alkaline counterpart, dibasic phosphate and bicarbonate, passes into the glomerular filtrate. If dibasic phosphate were reabsorbed by the renal tubules, the excreted monobasic phosphate could be titrated as acid in the urine (1). On the other hand, if bicarbonate were reabsorbed from the filtrate, and if the renal tubules were relatively impermeable to carbonic acid (in reality dissolved

1 Aided by grants from the United States Public Health Service and the John and Mary R. Markle Foundation. carbon dioxide), this acid could react with buffer salts and convert them into free titratable acid (4).

Similarly 2 hypothetical mechanisms depending on secretory activities of the tubular epithelium have been proposed as explanations of urinary acidification. The tubular secretion of molecular acid has been invoked to account for the conversion of the alkaline buffer components of the glomerular filtrate into their acid forms (5). On the other hand a pseudosecretory or ionic-exchange mechanism accomplishing the same end has been suggested (6). According to this latter view, hydrogen ions, formed within the tubular cells by the dissociation of carbonic acid, are exchanged for ions of fixed base bound by buffer salts in the tubular urine, thereby converting them into titratable buffer acids.

Recent studies on the dog $(7,8,9)$ have indicated that the induction of acidosis and the infusion of suitable buffers greatly facilitate the excretion of titratable acid. By measuring simultaneously the rate of filtration and reabsorption of phosphate and the rate of filtration of carbonic acid, it was demonstrated that the phosphate and carbonic acid filtration-reabsorption concepts are inadequate to explain the origin of urinary acid. Thus, by exclusion, it was shown that the tubular cells must add acid to the urine. Indirect evidence favored ionic exchange as the more reasonable of the 2 alternative mechanisms.

The present study of the mechanism of acidification of the urine by the normal human kidney was undertaken with 2 purposes in mind: first, to identify the nature of the mechanism; and second, to compare the acid excretory capacity of the human kidney with that of the dog. It has been found that the mechanisms are qualitatively similar in man and dog. Quantitatively, the capacity 
of the normal human kidney to excrete acid appears to exceed that of the dog.

\section{METHODS}

The 8 experiments forming the basis of this report, were performed on 4 healthy adult male subjects. Acidosis was induced by the ingestion of 20 grams of ammonium chloride on the day preceding the experiment. The salt was divided into 10 equal doses and was taken well diluted with water at hourly intervals throughout the day. No food was taken following the evening meal. Two glasses of water were ingested the morning of the experiment. Urines were collected by catheter and the bladder was washed with distilled water at the end of each collection period. Blood was drawn from 1 of the superficial veins of the forearm after soaking the limb in water at $47^{\circ}$ to $48^{\circ} \mathrm{C}$. for 5 to 7 minutes to render the composition of the venous blood essentially the same as that of arterial blood. For measurement of $\mathrm{pH}$ and carbon dioxide content, the blood was handled anaerobically (10). Analytical procedures for thiosulfate, phosphate, creatinine and urinary titratable acid and ammonia have been described in previous communications $(7,11)$.

Glomerular filtration rate was assessed by the thiosulfate clearance (12). Plasma thiosulfate concentrations were maintained between 30 and $40 \mathrm{mgm}$. per cent by continuous intravenous infusions. Both urine and plasma blanks were determined and appropriate corrections applied in all calculations. Neutral sodium phosphate 2 ( $\mathrm{pH} 7.4)$ was incorporated in the infusions and volume was adjusted by the addition of distilled water to render all solutions isotonic. The rate of infusion was varied between 3 and $13 \mathrm{ml}$. per minute to attain the desired plasma concentrations. No adverse reactions were observed from the infusion of phosphate; nausea and emesis followed the infusion of creatinine. At the highest plasma phosphate levels ( 6 to $7 \mathrm{mM}$ per liter) minimal signs of tetany were observed which subsided when the infusions were stopped.

\section{RESULTS}

\section{Experiments with phosphate}

Our plan of attack in identifying the nature of the renal mechanism for acidifying the urine has been to increase the rate of excretion of titratable acid by the induction of acidosis and by the infusion of large quantities of sodium phosphate. By measuring the quantities of phosphate filtered, reabsorbed and excreted and the quantity of carbonic acid filtered it has been possible to calculate exactly the maximum contribution which the hy-

${ }^{2}$ We are indebted to the William R. Warner Co. of New York for the preparation of a generous supply of ampoules of the sterile pyrogen-free sodium phosphate solution of $\mathrm{pH} 7.4$ used in this work.
THEORIES TO ACCOUNT FOR THE EXCRETION OF ACID URINE

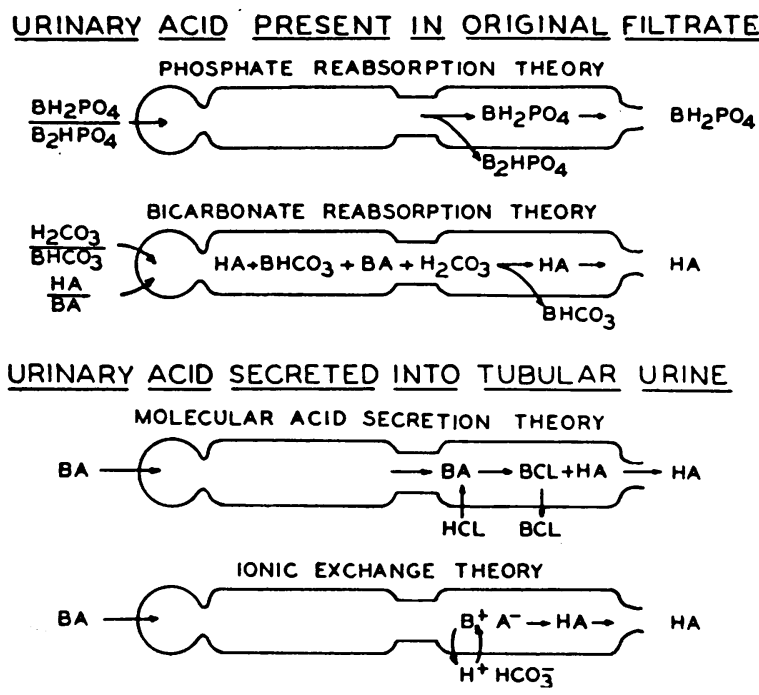

Fig. 1. For Explanation, See Text

pothetical phosphate and carbonic acid filtrationreabsorption mechanisms could possibly make to the excretion of titratable acid. Since monobasic phosphate and carbonic acid are the only acids present in significant amounts in a protein-free ultrafiltrate of plasma, it is obvious that if these substances cannot account for the observed rate of excretion of titratable acid, acid must be added to the urine by one or the other of the 2 active cellular transport mechanisms. Experiments presented below are conclusive in showing that tubular mechanisms are responsible for the addition of acid to the urine under our conditions of stress.

Experiment 1 in Table I illustrates both the character of the measurements and the relative significance of acidosis and plasma phosphate concentration in the experimental procedures employed. A moderately severe yet well compensated acidosis was induced by the ingestion of ammonium chloride. The extent of the acidosis is apparent in the plasma bicarbonate concentration of $14.2 \mathrm{mM}$ per liter, and the degree of compensation is evident both in the plasma $\mathrm{pH}$ of 7.37 and in the plasma carbonic acid concentration of $0.77,3$ shown in the first 2 periods of Experiment 1. In these

3 Normal values for R. F. P. are bicarbonate, 24 to 25 $\mathrm{mM}$ per liter; $\mathrm{pH}, 7.39$ to 7.41 ; carbonic acid, 1.17 to $1.23 \mathrm{mM}$ per liter. 


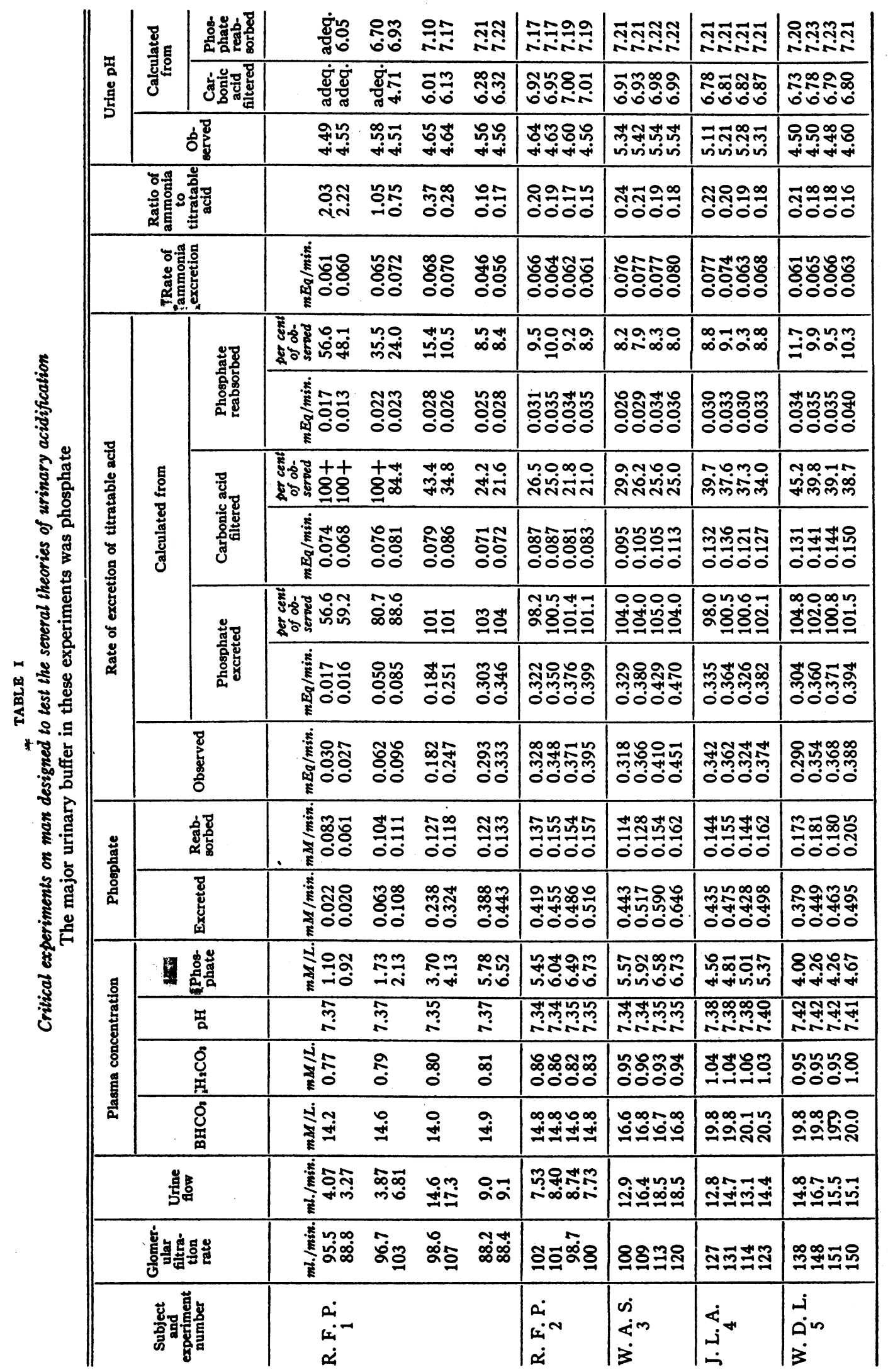


2 control periods the concentration of phosphate in the plasma ranged between 1.10 and $0.92 \mathrm{mM}$ per liter, or 0.00110 and $0.00092 \mathrm{mM}$ per milliliter. If these latter figures are multiplied by the glomerular filtration rate, one obtains the quantity of phosphate filtered per minute. The product of the urine concentration and urine flow yields the quantity of phosphate excreted per minute. The quantity of phosphate reabsorbed, equal to the difference between the quantities filtered and excreted, amounted initially to 0.083 and 0.061 $\mathrm{mM}$ per minute. Assuming this reabsorbed phosphate to be the dibasic form, the residual excess of monobasic phosphate left in the urine could account for the excretion of 0.017 and $0.016 \mathrm{mEq}$ of titratable acid per minute, or 56.6 to 48.1 per cent of that actually observed. Thus, acidosis alone rendered the phosphate reabsorption concept untenable in this experiment. In contrast, the filtration of carbonic acid is capable of explaining 100 per cent of the titratable acid eliminated in the 2 control periods.

In the succeeding 6 periods the plasma phosphate concentration was elevated in stepwise fashion to a maximum of $6.52 \mathrm{mM}$ per liter by the infusion of neutral sodium phosphate. The reabsorption of phosphate reached a maximum of 0.120 to $0.130 \mathrm{mM}$ per minute and the excess filtered, over and above this quantity reabsorbed, was excreted in the urine. The observed rate of excretion of titratable acid increased in linear fashion with the increased rate of excretion of phosphate despite the relatively constant rate of reabsorption. Accordingly, the phosphate reabsorption concept became progressively a less adequate explanation of urinary acidity, until in the final periods it could account for only 8 per cent of the observed acid. The quantity of carbonic acid filtered remained essentially constant throughout the experiment, varying only between 0.068 and $0.086 \mathrm{mEq}$ per minute. Accordingly from the fourth period on, the carbonic acid filtration concept likewise became an inadequate explanation of urinary acidity and in the final period could account for only 21.6 per cent of the acid excreted. It is apparent that titratable acid excretion can be increased to levels greatly above normal by increasing the quantity of phosphate excreted. In fact in the eighth period of Experiment 1 , a rate of excretion of $0.333 \mathrm{mEq}$ per min- ute is equivalent to the elimination of $4750 \mathrm{ml}$. of one-tenth normal acid per day. This is some 3 to 4 times the highest ever observed in diabetic ketosis. At plasma phosphate concentrations of 6 to $7 \mathrm{mM}$ per liter, the 2 hypothetical filtrationreabsorption mechanisms together can account for only one-third of the titratable acid excreted. Since these are the only significant sources of acid in the filtrate it is apparent that acid must be added to the tubular urine by some active cellular transport mechanism. It seems reasonable to us to assume that this cellular mechanism accounts for acid excretion under normal conditions as well as under conditions of acidosis and hyperphosphatemia, although it is impossible to identify its activity except by exceeding the limited acid excretory capacity of the 2 filtration-reabsorption mechanisms. We likewise feel from theoretical considerations discussed at length in a previous paper (7), that under no circumstances do phosphate reabsorption and carbonic acid filtration contribute significantly to the titratable acid of the urine.

With the information derived from this preliminary experiment, identical 4-period experiments were performed on 4 subjects, the results of which are presented in Table I. The extent of the acidosis varied considerably in the several subjects, as did the plasma concentration of phosphate. Variations in filtration rate, phosphate reabsorptive capacity and plasma carbonic acid concentration combined to alter the relative adequacies of the phosphate reabsorption concept and the carbonic acid filtration concept as explanations of the observed rates of excretion of titratable acid. In these latter 4 experiments phosphate reabsorption could account for only 8.0 to 11.7 per cent of the excreted acid. Carbonic acid filtration could account for only 21 to 40 per cent of the excreted acid. Both mechanisms together could account for only one-third to one-half of the acid. Thus, it is apparent that acid must be added to the urine by the renal tubules.

The columns in the center of the table headed rate of excretion of titratable acid calculated from phosphate excreted serve as a check on the accuracy of the chemical determinations, at least in those instances in which the rate of excretion of phosphate was high. In Experiments 2 to 5, values for titratable acid calculated from the $\mathrm{pH}$ 
of the urine and the rate of excretion of phosphate should agree with the observed values within reasonably small limits. The calculated values average $101.8^{4}$ per cent of the observed values. Actually the calculated values should average slightly less than 100 per cent, for there is a small moiety of non-phosphate titratable acid in the urine made up of creatinine and organic acid. However, the agreement is sufficiently close to warrant confidence in conclusions drawn from the more rigorous analysis of the data. In the initial 2 periods of Experiment 1, phosphate constituted between 50 and 60 per cent of the titratable acid; about half of the remainder was creatinine, about half was organic acid. As the excretion of phosphate and titratable acid increased, this nonphosphate moiety became a less and less significant proportion of the total. Whether it remained constant or diminished cannot be stated, because the range of experimental error of the determination at high phosphate levels is greater than this moiety.

The rate of ammonia excretion was followed in each experiment and averaged $0.066 \mathrm{mEq}$ per minute in the series presented in Table I. Although considerably increased over the normal, this rate of excretion is not high in comparison with that observed in diabetic ketosis, no doubt because the acidosis was not especially severe and was of short duration (less than 24 hours). In normal individuals and in patients in diabetic ketosis as well, the ratio of ammonia to titratable acid in the urine varies within limits of 1.0 and 2.5 (13). In the initial 2 periods of Experiment 1 the ratio amounted to 2.03 and 2.22 , well within the usual limits. Following the infusion of phosphate the ratio dropped progressively to a low of 0.16. This drop was brought about for the most part by the increase in rate of titratable acid excretion, not by diminution in ammonia elimination. Thus, the magnitude of this ratio in the presence of normal renal function is conditioned largely by the quantity and properties of the urinary buffers.

It is obviously possible to analyze the data in another way, namely in terms of the $\mathrm{pH}$ of the urine. The $\mathrm{pH}$ of the urine was calculated from

4 The $\mathrm{pK}^{\prime}$ for phosphate has been taken to be 6.80 . Obviously variation in this value in different urine samples is a possible source of error. the measured rate of buffer excretion assuming first that the only source of acid is filtered carbonic acid, and second that the source of acid is the excess monobasic phosphate remaining in the tubules following reabsorption of dibasic phosphate. The results of this analysis are presented in the last 3 columns of Table I. In order to perform this calculation it has been necessary to assume a $\mathrm{pK}^{\prime}$ for the non-phosphate moiety of the titratable acid. This has been taken to be that of creatinine, namely 4.97, an assumption which is only partially justified. Except in the initial 2 periods of Experiment 1, no serious error is introduced in the calculations if this assumed $\mathrm{pK}^{\prime}$ is erroneous. It is apparent from Experiment 1 that the phosphate reabsorption concept and the carbonic acid filtration concept are adequate to explain the $\mathrm{pH}$ of the urine only when the rate of buffer excretion is low. As the buffer content of the urine is increased by the infusion of phosphate, the deviations between observed and calculated urine $\mathrm{pH}$ values become large. It is interesting that 2 subjects, R. F. P. and W. D. L., were able to excrete urines containing large quantities of phosphate, yet of an acidity considerably greater than that assumed to be possible by the human kidney. The generally accepted limit of acidity of human urine is $\mathrm{pH} 4.7$ (14). Actually in 3 experiments on these 2 subjects all urines ranged in acidity between $\mathrm{pH} 4.48$ and 4.60 .

\section{Experiments with creatinine}

In order to test further the thesis that the urine is acidified by tubular secretory processes, we repeated the experiments substituting creatinine for phosphate as the buffer administered. Three experiments were performed on 3 different subjects. In the first experiment neither the quantity of creatinine administered nor the degree of acidosis was sufficiently great to yield conclusive results. The 2 more significant experiments are presented in Table II.

In Experiment 7 the acidosis was moderate; in Experiment 8, somewhat more severe; in both, the acidosis was well compensated. Sufficient creatinine was administered to raise the plasma concentration to 20 to $33 \mathrm{mgm}$. per cent and to effect the excretion of 0.296 to $0.466 \mathrm{mM}$ of this buffer per minute. The rate of elimination of endogenous phosphate varied between 0.016 and 


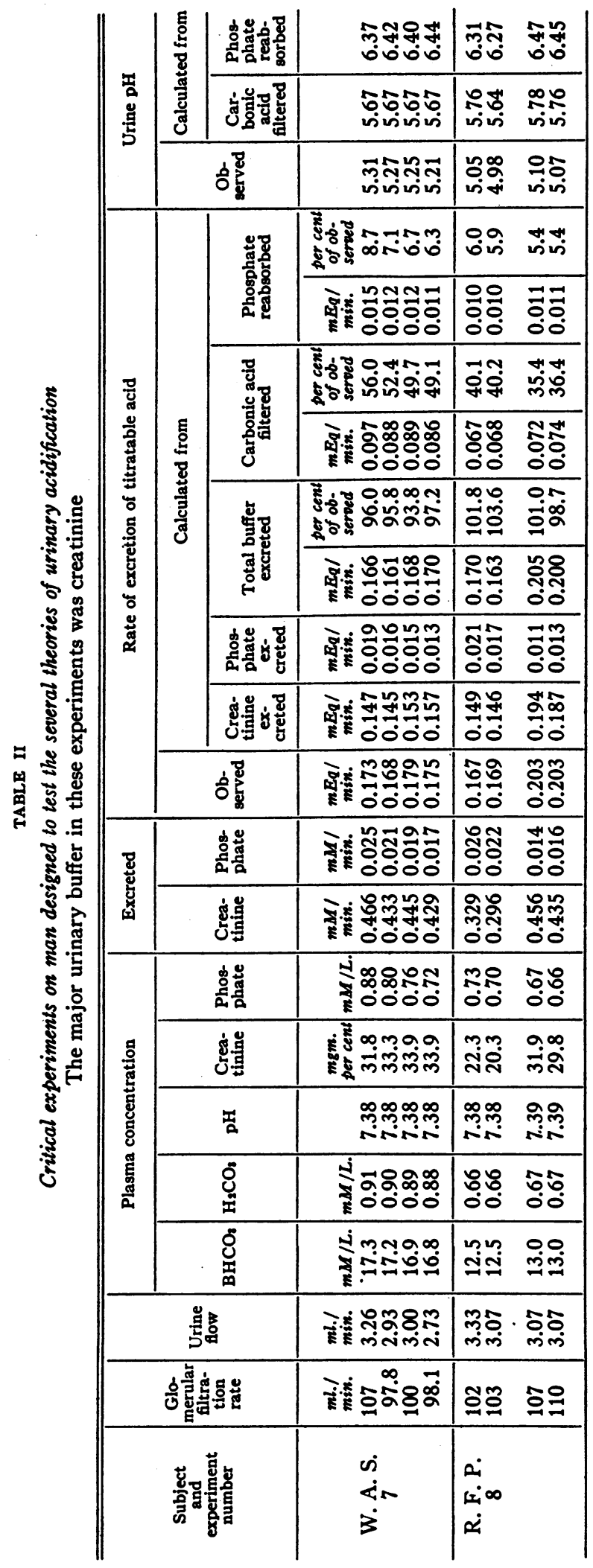


$0.026 \mathrm{mM}$ per minute. The values for titratable acid calculated from the urine $\mathrm{pH}$ and the sum of the creatinine and phosphate eliminated varied from 0.161 to $0.205 \mathrm{mEq}$ per minute. The observed rates of titratable acid excretion varied from 0.167 to $0.203 \mathrm{mEq}$ per minute, values much lower than those observed following phosphate administration. Nevertheless, calculated and observed values for titratable acid agreed within reasonable limits.

It is evident from the calculations of the maximum possible contributions of phosphate reabsorption and carbonic acid filtration to titratable acid excretion that neither concept adequately explains acidification of the urine. Thus, carbonic acid filtration could account for only 35 to 56 per cent, and phosphate reabsorption for only 5.4 to 8.7 per cent of the excreted acid. Similarly an analysis of the possible contributions of the 2 mechanisms to the excretion of urine of low $\mathrm{pH}$, indicates their essential incapacity to account for the degree of acidity actually obtained. Although the deficiencies of the 2 hypothetical filtration-reabsorption mechanisms are somewhat less striking in these experiments with creatinine than in the phosphate ones, they do confirm the thesis that active tubular processes must add acid to the urine. This confirmation is especially significant in view of the differences in the mechanisms by which the human kidney handles phosphate and creatinine: the former by filtration and reabsorption, the latter by filtration and secretion (15).

\section{DISCUSSION}

The data presented above are conclusive in demonstrating that under our experimental conditions the normal human kidney can excrete more acid per unit of time than could gain access to the urine in the glomerular filtrate. Therefore, by exclusion, acid must be added to the urine by some mechanism of active tubular transport. As pointed out above, this mechanism might be one of tubular secretion of molecular acid, or one of exchange of hydrogen ions formed within the renal tubular cells for ions of fixed base in the tubular urine. Our experimental methods cannot distinguish between these 2 possibilities. Indeed, as pointed out in previous work on the dog (7) the question is largely an academic one. If molecular acid, e.g., hydrochloric acid, were secreted by the renal tubules, the sodium chloride formed in its reaction with buffer salts would be largely reabsorbed further along the tubules. Therefore, in effect, an exchange of hydrogen ions for sodium ions would be brought about by secretion of molecular acid followed by reabsorption of neutral salt. Because the various salts in plasma, glomerular filtrate and urine are dissociated, it seems reasonable to assume that the kidney operates on ions rather than on molecular species, within the limitations imposed by the necessity for maintaining the electroneutrality of these several fluids. An ionic exchange mechanism would accomplish in 1 step the conservation of base and the excretion of acid, and incidentally satisfy requirements of electroneutrality.

Our experimental results on normal human subjects are so similar to those previously published on dogs that it seems legitimate to assume that the renal mechanisms are identical. One may therefore be permitted to extrapolate from certain experiments performed on dogs, too rigorous to be applicable to man, to fill in gaps in knowledge of the human renal mechanism. Obviously carbonic acid, formed by the hydration of carbon dioxide, is the only source of hydrogen ions available to the body in quantities sufficient to satisfy the demands imposed by the observed rates of urinary acid excretion. Kidney tissue contains large amounts of carbonic anhydrase, an enzyme which markedly increases the rate of hydration of carbon dioxide to carbonic acid (16). Sulfanilamide, an inhibitor of carbonic anhydrase (17), when present in the plasma in high concentration (30 to $80 \mathrm{mgm}$. per cent) greatly reduces or abolishes the excretion of titratable acid in the $\operatorname{dog}(7)$. From these facts it was concluded that carbonic anhydrase is an essential link in the tubular ionic exchange mechanism, and that its role is to speed the hydration of carbon dioxide to carbonic acid within the cells of the renal tubules. The dissociation of this carbonic acid provides hydrogen ions for exchange with base bound by buffers of the tubular urine. The base in association with bicarbonate ions is then returned to the renal venous blood. We feel that these conclusions regarding the nature of the tubular mechanism in so far as they are valid for the dog, are 
equally applicable in man, especially in view of the disturbances in acid-base balance described in the early clinical use of sulfanilamide (18). Indeed we feel that one of the most significant aspects of the present investigation lies in its justification of the use of the dog for analysis of problems of acid-base regulation.

In a quantitative sense, we feel that the capacity of the human kidney to excrete acid probably exceeds that of the dog. This statement may not be generally valid for it is based only upon a comparison of 4 healthy young adults with 4 healthy mongrel dogs. But the fact that the results are so consistent seems to justify this tentative statement. In experiments on dogs (7) similar to those reported above, the highest rate of titratable acid excretion which was observed amounted to $0.380 \mathrm{mEq}$ per minute. This was attained at a plasma phosphate level of $9.84 \mathrm{mM}$ per liter and at a rate of excretion of phosphate of 0.613 $\mathrm{mM}$ per minute. In attaining this rate of acid excretion, the kidney elaborated a urine of $\mathrm{pH}$ 6.07 ; in essence the kidney could establish a concentration gradient for hydrogen ions between tubular urine and plasma of somewhat less than 20 to 1 . The 4 human subjects excreted from 0.374 to $0.451 \mathrm{mEq}$ of titratable acid at plasma phosphate concentrations which were substantially lower than those of the dogs. Furthermore, at comparable rates of phosphate excretion, urines of much lower $\mathrm{pH}$ (4.48 to 5.54) were elaborated by the human subjects. Thus the human kidney can transfer the same or larger quantities of hydrogen ions against higher concentration gradients. Subjects W. A. S. and J. L. A. could establish a gradient of roughly 100 to 1 while transferring approximately $0.400 \mathrm{mEq}$ of hydrogen ions per minute; subjects R. F. P. and W. D. L. could establish a gradient of 800 to 1 while transferring equivalent quantities of hydrogen ions. The difficulties of comparing acid excretory capacity in dog and man not only lie in differences in body and kidney size, but likewise in the fact that in neither dog nor man was any absolute maximum for acid excretion attained. Doubtless further increases in the rate of phosphate excretion in both forms would have led to further increases in acid excretion.

\section{SUM MARY}

In a series of 8 experiments on 4 healthy human subjects it was found that the induction of acidosis by the ingestion of ammonium chloride and the promotion of buffer excretion by the infusion of phosphate or creatinine greatly increased the rate of excretion of titratable acid. Simultaneous measurement of the rate of filtration, reabsorption and excretion of mono- and dibasic phosphate and carbonic acid demonstrated that the quantity of acid excreted far exceeded that which entered the urine in the glomerular filtrate. Therefore, acid must have been added to the filtrate as it passed along the nephron by some mechanism of active transport resident in the renal tubular cells. It is suggested that this addition of acid is effected by the exchange of hydrogen ions formed within the tubular cells for ions of fixed base in the tubular urine. Carbonic acid is doubtless the intracellular source of hydrogen ions.

This mechanism for acid excretion in the human kidney is qualitatively similar to that previously described in the dog. Quantitatively, the human kidney has a greater acid excretory capacity than the dog kidney.

\section{BIBLIOGRAPHY}

1. Peters, J. P., and Van Slyke, D. D., Quantitative Clinical Chemistry, Vol. 1, Interpretations. Williagms and Wilkins Co., Baltimore, 1932.

2. Stillman, E., Van Slyke, D. D., Cullen, G. E., and Fitz, R., Studies on acidosis. VI. The blood, urine and alveolar air in diabetic acidosis. J. Biol. Chem., 1917, 30, 405.

3. Henderson, L. J., A critical study of the process of acid excretion. J. Biol. Chem., 1911, 9, 403.

4. Sendroy, J., Jr., Seelig, S., and Van Slyke, D. D., Studies on acidosis. XXIII. The carbon dioxide tension and acid-base balance of human urine. $\mathrm{J}$. Biol. Chem., 1934, 106, 479.

5. Macallum, A. B., and Campbell, W. R., The secretion of acid by the kidney. Am. J. Physiol., 1929, 90, 439.

6. Smith, H. W., The Physiology of the Kidney. Oxford University Press, New York, 1937.

7. Pitts, R. F., and Alexander, R. S., The nature of the renal tubular mechanism for acidifying the urine. Am. J. Physiol., 1945, 144, 239.

8. Pitts, R. F., and Lotspeich, W. D., Factors governing the rate of excretion of titratable acid in the dog. Am. J. Physiol., 1946, 147, 481.

9. Pitts, R. F., The renal regulation of acid-base balance with special reference to the mechanism for acidifying the urine. Science, 1945, 102, 49 and 81. 
10. Pitts, R. F., and Lotspeich, W. D., Bicarbonate and the renal regulation of acid-base balance. Am. J. Physiol., 1946, 147, 138.

11. Pitts, R. F., and Lotspeich, W. D., Use of thiosulfate clearance as a measure of glomerular filtration rate in acidotic dogs. Proc. Soc. Exper. Biol. and Med., 1947, 64, 224.

12. Newman, E. V., Gilman, A., and Philips, F. S., The renal clearance of thiosulfate in man. Bull. Johns Hopkins Hosp., 1946, 79, 229.

13. Van Slyke, D. D., Linder, G. C., Hiller, A., Leiter, L., and McIntosh, J. F., The excretion of ammonia and titratable acid in nephritis. J. Clin. Invest., 1926, 2, 255.
14. Henderson, L. J., and Palmer, W. W., On the intensity of urinary acidity in normal and pathological conditions. J. Biol. Chem., 1913, 13, 393.

15. Shannon, J. A., The renal excretion of creatinine in man. J. Clin. Invest., 1935, 14, 403.

16. Davenport, H. W., and Wilhelmi, A. E., Renal carbonic anhydrase. Proc. Soc. Exper. Biol. and Med., 1941, 48, 53.

17. Davenport, H. W., The inhibition of carbonic anhydrase by thiophene-2-sulfonamide and sulfanilamide. J. Biol. Chem., 1945, 158, 567.

18. Southworth, H., Acidosis associated with the administration of para-amino-benzene-sulfonamide (prontylin). Proc. Soc. Exper. Biol. \& Med., 1937, $36,58$. 